\title{
Random Lasers: Characteristics, Applications and Some Research Results
}

\author{
Dinh Van Hoang ${ }^{1}$, Nguyen Thi Phuong ${ }^{2}$, Nguyen Van Phu ${ }^{3}$ \\ ${ }^{1}$ Hanoi National University, Vietnam \\ ${ }^{2}$ Hanoi Agricultural University, Vietnam \\ ${ }^{3}$ Vinh University, Vietnam
}

(Received: 29 June 2010; accepted: 13 September 2010)

\begin{abstract}
The random laser differs from other types of laser in that its cavity is formed not by mirrors but by multiple scattering in a disordered gain medium. Over the course of ten years, random lasers have been attentive by many researchers in the world because their characteristics can lead to unique applications. This report presents the mechanism of random laser operation, classification, characteristics and prediction of different applications. Some research results of authors about random laser have also been noted in report.
\end{abstract}

Key words: random laser, multiple scattering, disordered gain medium

\section{INTRODUCTION}

As we know, the most common laser cavity is the Fabry -Perot made of two mirrors (one of which is partially transmitting), that place each other on either side of the gain medium (Fig. 1).

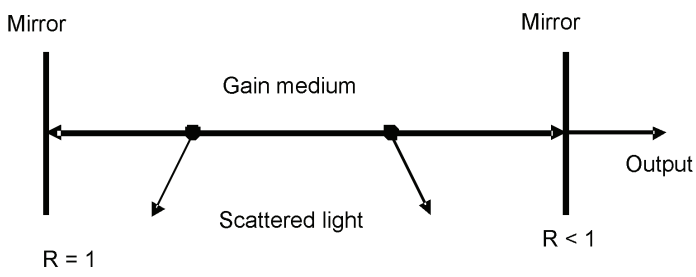

Fig. 1. Fabry-Perot cavity in a typical laser

Light that is bounced back and forth between two mirrors is amplified each time it passes through the gain medium. Light can leave the cavity through the partially transmitting mirror. On the other hand, light that remains in the cavity interferes constructively after traveling a round trip between the mirrors and returning to its original position. When the optical amplification is large enough to compensate the loss caused by mirror leakage and material absorption, etc., lasing oscillations occur at cavity resonant frequencies. The laser light transmitted through the partially reflecting mirror has well defined frequency, good direc- tionality and a high degree of coherence. However, in this case, inside the cavity there are also scatterers and the light can be scattered in other directions. This results in additional loss and increasing the lasing threshold. Therefore, in conventional laser optical scattering is considered detrimental and we have to minimize the amount of scattering in laser cavities.

What happens when the scattering is strong? In this case, surprisingly, one can receive lasing oscillations. In fact, let us consider a disordered medium that contains a number of scattering centers (see Fig. 2).

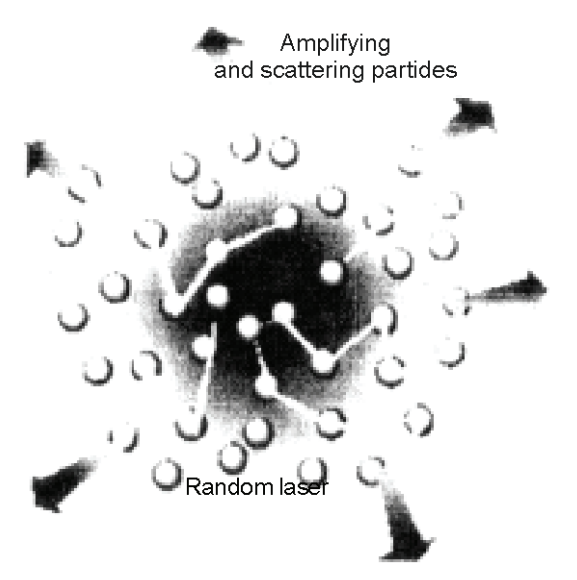

Fig. 2. A disorded medium with scattering centers 
Light is scattered many times before it escapes the gain medium. The multiple scattering increases the dwell time of light, enhancing light amplification. In this scenario, mirrors are not needed to trap the light in the gain medium because scattering can do the job on its own. Thus strong scattering in a disordered media can give us laser operation and we receive random laser. The word "random" has been used to describe lasers that operate on the basics of these properties.

In short, the random laser differ from other conventional types of laser in that its cavity is formed not by mirrors but by multiple scattering in a disordered gain medium.

In other words, a random laser is a non-conventional laser whose feedback mechanism is based on disorder - induced light scattering. Afterwards we present the classification, some characteristics and application of random lasers.

\section{CLASSIFICATION}

Today one classifies two types of random laser: Random laser with incoherent feedback and random laser with coherent feedback.

\section{Random laser with incoherent feedback}

Let us consider one Fabry-Perot cavity in which one mirror is replaced by a scattering surface. Light in this cavity undergoes multiple scattering. Since it is scattered every time, its direction is changed and it does not return to its original position after one roundtrip. In such a cavity the spatial resonances of electromagnetic field are absent and the dwell time of light is not sensitive to frequency. In this case the feedback is used simply to return part of energy or photons to the gain medium, i.e. it is energy or intensity feedback.

Thus, in a laser with incoherent feedback or nonresonant feedback the only resonant element is the amplification line of the gain medium. The mean frequency of emission does not depend on the dimensions of laser cavity but only on the center frequency of the amplification line.

\section{Random laser with coherent feedback}

The main difference between random laser with incoherent feedback and the one with coherent feedback is that in the coherent random laser the light can return to its origin position of disordered medium from which it was scattered. It means that light can form a closed loop. This process is called recurrent light scattering (see loop in Fig. 2).

For each loop, when the amplification of light along the loop exceeds the loss, lasing oscillation occurs and each loop can serve as cavity analogous to ring cavity of conventional laser. Therefore, if scattering light forms some various loops, disordered medium lasing some determined frequencies and we receive some modes of coherent random laser.
In this scenario the behavior of the coherent random laser is very similar to that of a conventional laser.

The significant difference between the coherent random laser and the normal laser is that the random laser has not directional output. In other words, the output is multidirectional and the direction of each individual mode is different.

\section{CHARACTERISTICS OF RANDOM LASER}

It is difficult to indicate significant characteristics of random laser because this domain has not been studied long, the operation mechanism of random laser remains not clear, the structure of disordered medium is very diverse, etc. However, we can reveal some obvious characteristics as follows:

1. The output of random laser modes is multidirectional, which means the modes have spatial distribution. The observation along different directions can result in different recognition about random laser modes.

2. When the pump intensity increases the output of modes and the number of modes increase too. While spectral narrowing of the emission lines occurred (see Fig. 3).

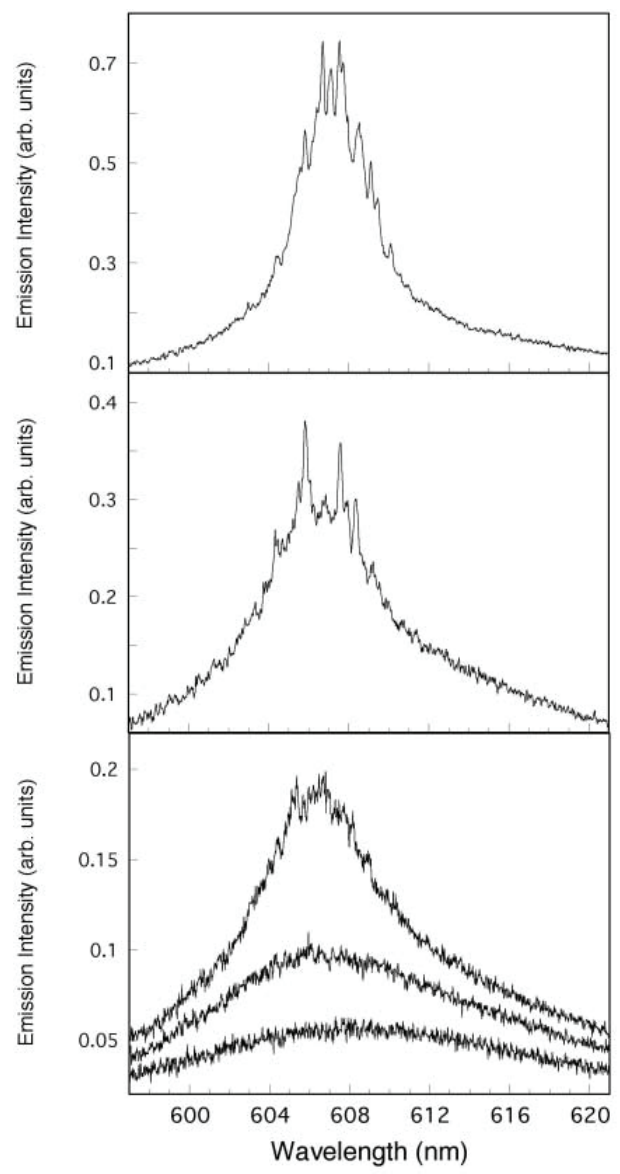

Fig. 3. Emission Intensity of the laser output 
3. There is a mode competition between the random laser modes that results in the photon hopping effect, i.e. the hop of photon from one mode to the other modes and the field interaction effect between side modes.

\section{APPLICATIONS}

1. Random laser can be lasing in a wide spectral regime from ultra short wave ( $\gamma$-ray, $X$-ray) to optical and radio wave. The construction of random laser lasing $\gamma$-ray, $X$-ray, is certainly cheaper and easier than the one of the conventional laser.

2. Random laser can be used in document encoding, material labeling where its frequencies represent the signature of subjects.

3. Multidirectional output of a random laser can be used in displays because a thin layer of random medium doped with emitter can be used to coat an arbitrarily shaped display panel. Moreover, the fast switching on and off of random laser can be used also to create high speed display.

4. In the area of search and rescue the painted on laser of random laser may provide a rugged and low cost method of identification for downed ships, aircraft and satellites.

5. In the medical area, the random laser has potential application to photo dynamic therapy and tumor detection.

6. The micro random laser may play the crucial role of an active element or miniature light source in integrated photonic circuit. It can also be used to monitor the flow of liquids (for example blood) by adding a small number of nanoparticale clusters to the liquid and detecting their laser emission over a large flow distance.

\section{SOME RESEARCH RESULTS}

In the process in random laser, our groups concentrate to the study about the operation mechanism of random laser. The appearance of modes reflects transforming processes in disordered medium in random laser. Especially, the interaction of modes also reflects the interaction of eigenstates of a random system. Hence, knowledge about it is necessary to understanding the escape channels for photons in an eigenstate that is important to the localization theory. Therefore, questions posed here are:

- How do the random laser modes appear?

- What is the interaction between these modes?

- What is the relation between the transformation of modes and the phenomena exhibiting in a disordered medium?
To answer these questions, let us consider a system of random laser lasing two, three modes and so on. Below are presented basic equations and the main research results.

\section{Basic equations}

1.1. For the case lasing two modes, we have:

$$
\begin{aligned}
& \frac{d n_{1}}{d t}=\alpha_{1} n_{1}-\gamma_{1} n_{1}-\theta_{12} n_{1} n_{2}+\gamma_{21} n_{2}, \\
& \frac{d n_{2}}{d t}=\alpha_{2} n_{2}-\gamma_{2} n_{2}-\theta_{21} n_{2} n_{1}+\gamma_{12} n_{1},
\end{aligned}
$$

where $\alpha_{l}, \gamma_{l}(i=1,2)$ designed gain and loss coefficients, $\theta_{12}, \theta_{21}-$ field coupling coefficients; $\gamma_{12}, \gamma_{21}-$ photon hopping coefficients indicating the leakage of photon from one to another mode and inverse. Because the loss of mode photon in random laser for most material is generally proportional to photon densities $n_{i}\left(\right.$ i.e $\left.\gamma_{i}=\beta_{i} n_{i}\right)$, therefore, Eqs. (1) and (2) become:

$$
\begin{aligned}
& \frac{d n_{1}}{d t}=\alpha_{1} n_{1}-\beta_{1} n_{1}^{2}-\theta_{12} n_{1} n_{2}+\gamma_{21} n_{2}, \\
& \frac{d n_{2}}{d t}=\alpha_{2} n_{2}-\beta_{2} n_{2}^{2}-\theta_{21} n_{2} n_{1}+\gamma_{12} n_{1} .
\end{aligned}
$$

\subsection{For the case lasing three modes}

Supposed mode $n_{2}$ lies in the middle of two other modes. We have:

$$
\begin{gathered}
\frac{d n_{1}}{d t}=\alpha_{1} n_{1}-\beta_{1} n_{1}^{2}-\theta_{12} n_{1} n_{2}+\gamma_{21} n_{2} \\
\frac{d n_{2}}{d t}=\alpha_{2} n_{2}-\beta_{2} n_{2}^{2}-\left(\theta_{21} n_{1}+\theta_{23} n_{3}\right) n_{2}+\gamma_{12} n_{1}+\gamma_{23} n_{3} \\
\frac{d n_{3}}{d t}=\alpha_{3} n_{3}-\beta n_{3}^{2}-\theta_{32} n_{3} n_{2}+\gamma_{32} n_{2}
\end{gathered}
$$

\section{Solution and discussions}

To solve these equations above we used the numerical method. The values of coefficients in equations (i.e. dynamic laser parameters) are taken from experiment and Refs. [10-13] as follows:

- For two modes case:

$$
\begin{gathered}
\alpha_{1}=1.1 \mathrm{~s}^{-1} ; \quad \beta_{1}=0.4 \mathrm{~cm}^{3} \mathrm{~s}^{-1} ; \\
\theta_{12}=0.8 \mathrm{~cm}^{3} \mathrm{~s}^{-1} ; \gamma_{12}=0.35 \mathrm{~s}^{-1} ; \\
\alpha_{2}=0.9 \mathrm{~s}^{-1} ; \beta_{2}=0.3 \mathrm{~cm}^{3} \mathrm{~s}^{-1} ; \\
\theta_{21}=0.7 \mathrm{~cm}^{3} \mathrm{~s}^{-1} ; \gamma_{21}=0.35 \mathrm{~s}^{-1} .
\end{gathered}
$$

- For two three modes case:

$$
\begin{aligned}
\alpha_{1} & =0.9 \mathrm{~s}^{-1} ; \beta_{1}=0.4 \mathrm{~cm}^{3} \mathrm{~s}^{-1} ; \\
\theta_{12} & =0.8 \mathrm{~cm}^{3} \mathrm{~s}^{-1} ; \gamma_{12}=0.4 \mathrm{~s}^{-1} ;
\end{aligned}
$$




$$
\begin{aligned}
\alpha_{2} & =1.3 \mathrm{~s}^{-1} ; \beta_{2}=0.3 \mathrm{~cm}^{3} \mathrm{~s}^{-1} ; \\
\theta_{21}=\theta_{23} & =0.7 \mathrm{~cm}^{3} \mathrm{~s}^{-1} ; \gamma_{21}=\gamma_{23}=0.4 \mathrm{~s}^{-1} ; \\
\alpha_{3} & =0.9 \mathrm{~s}^{-1} ; \beta_{3}=0.4 \mathrm{~cm}^{3} \mathrm{~s}^{-1} ; \\
\theta_{32} & =0.8 \mathrm{~cm}^{3} \mathrm{~s}^{-1} ; \gamma_{32}=0.4 \mathrm{~s}^{-1} .
\end{aligned}
$$

Using Matlab language with $n_{i}(0)=0.8$, we received the curves of $n_{i}(t)$ as present in Figs. $4 \mathrm{a}$ and $4 \mathrm{~b}$, respectively for the case of two modes and three modes.

a)

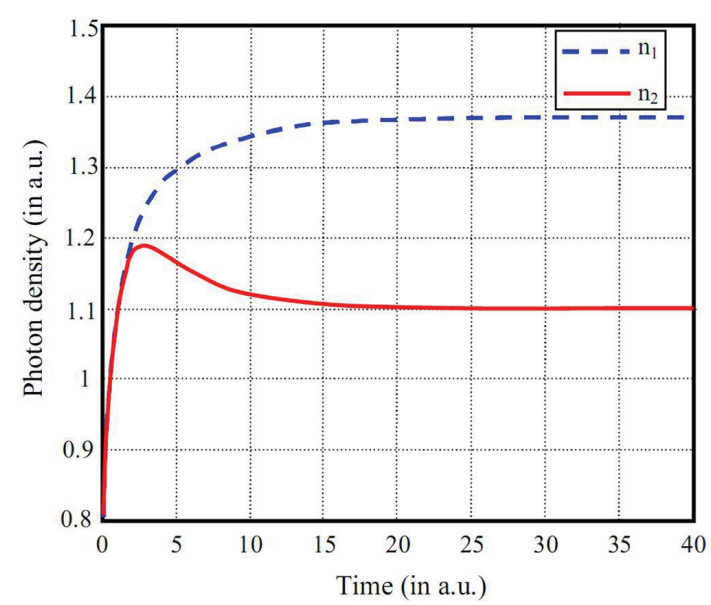

Fig. 4a. The curves of $n_{i}(t)$ for two modes

b)

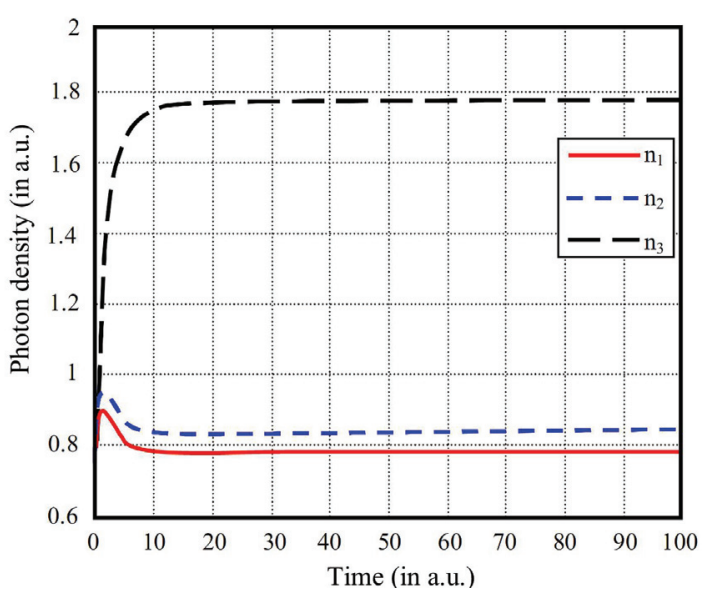

Fig. 4. The curves of $n_{i}(t)$ for three modes

From the figures we see that:

- Appearance process of modes in random laser composes three periods: development, competition or interaction between modes and saturation period.

- Time interval for each period certainly depends on material, manufacture process, structure of disordered medium in random laser.

- Characteristics of random laser modes like intensity or output of modes, the saturation values of photon densities will be transformed, when coefficients in equations vary. This lets us examine the influence of dyna- mic laser parameters (i.e. coefficients in equations) on curves $n_{i}(t)$. The detail study of this problem has been presented in our articles $[14,15]$ and we can resume the following:

- When the gain or amplification coefficient $\alpha_{i}$ changes, the curves describing the operation of laser and the values of saturation photon densities of mode vary. The increase of gain coefficient of any mode results in the augmentation of saturation photon density of this mode and the diminution of the one of other modes. Inverse situation exists when gain coefficient decreases. The change of any mode always influences mainly on the side modes. This reveals effect of near interaction and it is distinguished in operation of multimode random laser.

- The increase or decrease of loss coefficients $\beta_{i}$ of any mode makes the diminution or augmentation of mode photon density value $n_{i}^{S}$ of this mode. The transformation of curves $n_{i}(t)$ due to the variation of loss coefficient always is inverse to the one due to the change of gain coefficient.

- At very small loss coefficient (or at vary big gain coefficient) of any mode, only this mode is generated. The operation of multimode random laser becomes the one of single mode laser. This result gives us a possibility to use random laser in many different applications.

\section{CONCLUSION}

In this Symposium we would like to introduce a new type of laser that is the random laser. The research in our country is still new and not broad. The study results are only in the initial stage.

We hope that with unique and specific characters, the random laser will take a worthy place in application domain of laser in general.

\section{References}

[1] H. Cao, Waves in random laser media 13, 1-39 (2003).

[2] H. Cao, Optics and photonics news 24 (2005).

[3] H. Cao et al., Appl. Phys. Lett. 73, 3656 (1998).

[4] H. Cao et al., Phys. Rev. B 59, 15107 (1999).

[5] H. Cao et al., Phys. Rev. Lett. 82, 2278 (1999).

[6] H. Cao et al., Phys. Rev. Lett. 84, 584 (2000).

[7] D. Wiersma, Nature 406, 132 (2000).

[8] A. Mitra, R.K. Thareja, Mod. Phys. Lett. B 23, 1075 (1999).

[9] R.K. Thareja, A. Mitra, Appl. Phys. B 71, 181 (2000).

[10] V.M. Markusev et. al., Sov. J. Quant. Electronics, T 16, 281 (1986). 
[11] N.M. Lawandy et al., Nature T 368, 436 (1994).

[12] X. Jiang, C.M. Soukoulis, Phys. Rev. Lett. 85, 70 (2000).

[13] X. Jiang et al., Phys. Rev. B 69, 104202 (2004).
[14] Dinh Van Hoang, Mai Hong Hanh, VNU J. of Science, Math.-Phys. 22 (2), 65 (2006).

[15] Dinh Van Hoang, Mai Hong Hanh, VNU J. of Science, Math.-Phys. 23 (3), 139 (2007).
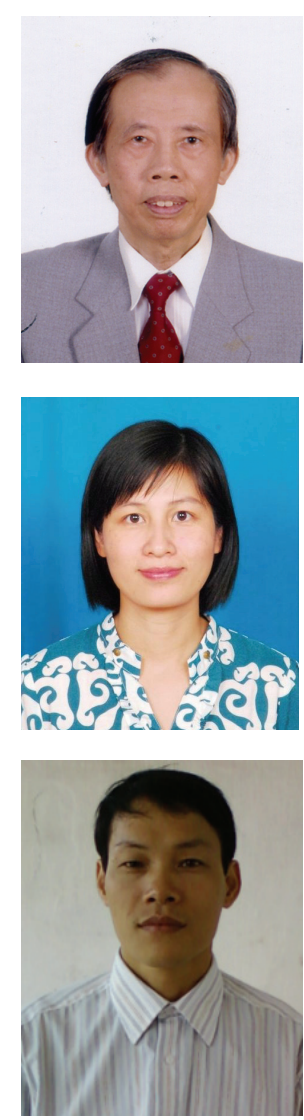

DinH VAN HoANG received Candidate nayok degree in 1965 from Byelorussia National University at Minsk city in Optics and Spectroscopy, received Doctor of Science (Doctor Habil.) in 1982 from Academy of Science of ancient DDR at Berlin city in Laser Physics. His field of interest covers laser physics, optics and Spectroscopy, Nonlinear Optics, Optical Bistability. He is professor at Faculty of Physics of Hanoi National University.

Nguyen Thi Phuong has graduated in Physics in 1998 and received the MSC degree in 2007 in Laser Physics at Hanoi National University. Now she is a teacher at Hanoi Agriculture University.

NguYen Van Phu was born in Nghe An provice, Vietnam. He completed his MSc study in 2000 at Vinh University under supervision of Prof Nguyen Huy Cong. Since 2003 he jointed to group led by professor Dinh Van Hoang to work as PhD student. Here he did studies on a new development of two and three-section DFB lasers. His field of interest concern the theory of laser and nonlinear optics. 